\title{
Compartmentalization of Anterogradely and Retrogradely Transported Organelles in Axons and Growth Cones from Chick Optic Tectum
}

\author{
T. P. O. Cheng and T. S. Reese \\ Laboratory of Neurobiology, IRP, NINCDS, at The Marine Biological Laboratory, National Institutes of Health, Woods Hole, \\ Massachusetts 02543.
}

Previous work suggests that organelles contacting microtubules in axons are in fast transport. Here, we examine the distribution of organelles contacting microtubules in growing axons and growth cones from chick optic tectum. Five axon segments, each $10 \mu \mathrm{m}$ long, and 4 entire growth cones were reconstructed from serial electron micrographs of quick-frozen, freeze-substituted chick optic tectum. Organelles contacting microtubules in axons are evenly distributed along all microtubules. Smaller organelles, presumably in anterograde transport, are enclosed in fascicles of microtubules, while larger organelles in retrograde transport lie outside the fascicles. In contrast, organelles contacting microtubules are prevalent only in the most proximal parts of the growth cone, before the microtubule fascicles splay out more distally. The distance between noncontacting organelles and microtubules also becomes progressively greater, reaching a maximum in the mid- and more distal region of the growth cone. Contacts with microtubules of both the smaller, presumably anterogradely transported organelles, as well as the larger, presumably retrogradely transported organelles, abruptly become less frequent in the proximal midregion of the growth cone. It is therefore of possible significance in stopping and starting microtubule-based organelle transport that microtubules change from a straight to an undulating configuration in the midregion of the growth cone. The decrease in organelle binding to microtubules at the demarcations between the straight and undulating microtubule segments may depend on proteins or other local factors as well as the splaying out of the microtubule bundles.

Various organelles-coated and uncoated vesicles, spherical and flat vacuoles, and lumenless membrane disks-are believed to be involved in local recycling of the growth cone plasmalemma in the distal growth cones from intact chick optic tecta (Cheng and Reese, 1985a, b, 1987). Vesicles and vacuoles, as well as other types of organelles, are also believed to be recycled into or out of growth cones by fast axonal transport (Berlinrood et al., 1972; Breuer et al., 1975; Leestma, 1976). Larger vacuoles carry specific materials out of mature nerve terminals back to the cell body (Kristenson and Olsson, 1971; Hendry et al., 1974;

\footnotetext{
Received June 8, 1987; accepted Nov. 6, 1987.

We wish to thank John C. Murphy for his help with all aspects of the photography and Eric Frank for his valuable discussion that led us to compare model Poisson distributions with actual distribution of organelles contacting axonal microtubules

Correspondence should be addressed to T. P. O. Cheng at the above address. 0270-6474/88/093190-10\$02.00/0
}

LaVail and LaVail, 1974; Stoekel and Thoenen, 1975; Kristenson, 1977; Tsukita and Ishikawa, 1980); this retrograde transport appears to occur in the tips of growing neurites as well (Berlinrood et al., 1972; Breuer et al., 1975; Bunge, 1977).

Local recycling of the growth cone plasmalemma (Cheng and Reese, 1987) clearly cannot by itself account for the net gain of plasma membrane during elongation of the axons. Additional membrane from other sources would be needed for the expansion of the chick tectal growth cone. Our present working hypothesis is that some of the anterogradely transported organelles are used to augment the pool of membranes engaged in local recycling of the filopodial plasmalemma (Cheng and Reese, 1987) and that the membrane addition steps in the local membrane recycle provide the means of adding plasma membrane for axonal growth (Bray et al., 1978; Feldman et al., 1981; Pfenninger and Maylie-Pfenninger, 1981).

Recent video microscopic studies of fast axonal transport in extruded squid axoplasm indicate that a single microtubule can even support movement of organelles in both directions (Cooper and Smith, 1974; Allen et al., 1982; Brady et al., 1982; Hayden, et al., 1983; Schnapp et al., 1985). Functional coupling between translocating organelles and microtubules appears to involve close physical juxtaposition between these 2 elements (Raine et al., 1971; Allen, 1975; Martz et al., 1984; Miller and Lasek, 1985; Schroer et al., 1985; Vale et al., 1985a, b; Kachar et al., 1987), which could best be preserved by quick-freezing and freeze-substitution techniques (see also Schnapp and Reese, 1982). Therefore, these techniques were used to determine which organelles are contacting microtubules in axons and growth cones, on the assumption that organelles contacting microtubules are engaged in fast axonal transport.

Assuming that small organelles move anterogradely, away from the cell body, and that large organelles move retrogradely, toward the cell body (Smith, 1980; Miller and Lasek, 1985), we analyze in series of thin sections the distributions of organelles in contact with microtubules in order to determine whether organelles in anterograde (vesicles with diameters $<150 \mathrm{~nm}$ ) and retrograde (vacuoles with diameters $>150 \mathrm{~nm}$ ) transport are located preferentially in the vicinity of the microtubule fascicles in axons and growth cones.

We found that vesicles and vacuoles have different preferred positions in relation to the fascicles of neuritic microtubules. Our measurements of the distributions of organelles along growth cone microtubules also indicated that vesicles in anterograde transport appear to be unloaded from microtubules in a specific region of the tectal growth cone which is slightly proximal 
to the midregion where vacuoles destined for retrograde transport are loaded onto microtubules. Very few of the vesicles and vacuoles in the distal regions of the growth contact microtubules; the high concentration of these distal unattached organelles is consistent with our conclusion that these organelles, the ones that have been shown to be involved in local recycling of the growth cone plasmalemma (Cheng and Reese, 1985a, b; 1987), are not in transport along microtubules.

\section{Materials and Methods}

Details of specimen preparation, techniques for computer-assisted serial reconstruction, and related procedures have been described elsewhere (Cheng and Reese, 1987).

Preparation of specimens. Chicken embryos at $6 \frac{1 / 4-61 / 2}{d}$ of incubation were placed in minimum essential medium (GIBCO Lab, Grand Island, NY) for removal of optic tecta. The dissected tecta were slam-frozen within approximately $1 \mathrm{~min}$, as described previously (Cheng and Reese, 1985a). The frozen samples were then freeze-substituted in a mixture of osmium tetroxide $(5 \%)$ and acetone for $17 \mathrm{hr}$ at $-78^{\circ} \mathrm{C}$, warmed to $0^{\circ} \mathrm{C}$, rinsed with a precooled acetone-methanol mixture followed by $100 \%$ methanol, and finally stained with $20 \%$ uranyl acetate in absolute methanol for $5 \mathrm{hr}$ at $0^{\circ} \mathrm{C}$. They were then rinsed with methanol, warmed to room temperature, and embedded in Araldite for routine processing for serial sectioning (Cheng and Reese, 1985a).

Computer-assisted serial reconstruction. Ribbons of approximately 250 serial sections were subdivided into 10 short ribbons and each ribbon was mounted on a Formvar-coated copper slot grid. After heavymetal staining, the sections were air-dried and then examined in a JEOL $200-\mathrm{CX}$ electron microscope at $120 \mathrm{kV}$. During printing of a series, each microtubule array was aligned in overall best-fit registration with respect to the previous section (Cheng and Reese, 1985a). The continuity of microtubules was determined by examining the micrographs printed at a final magnification of $120,000 \times$. The positions of microtubules within the outlines of the neurites or growth cones were then recorded with a computer-driven digitizer. After completion of a series, the digitized outlines were built up into 3-dimensional images of the neurite or growth cone segment containing composite images of most of the microtubules in a neurite or a growth cone. The data acquisition and subsequent graphic display were done with a Tektronix Graphic Tablet 4956 and Graphic System 4054 (Tektronix, Beaverton, OR 97075).

For planimetric measurements, the perimeter of each profile was digitized consecutively 7-10 times and the average cross-sectional area was multiplied by the section thickness $(0.1 \mu \mathrm{m})$ to yield the volume within each section. The final volumes of neurite and growth cone segments were obtained by totaling the volumes of single sections. The diameters of vesicles and vacuoles, as well as their distances from microtubules, were measured on micrographs of serial sections printed at a final magnification of $120,000 \times$.

Statistics. One hundred serial sections from each of 5 neurite segments, $10 \mu \mathrm{m}$ long, as well as approximately 250 serial sections from each of 4 complete growth cones were reconstructed. To determine whether all microtubules in neurites transport organelles, all of the microtubules in the 5 reconstructed neurites were pooled and the number of microtubule-contacting organelles counted. The expected numbers of microtubules with different numbers of attached organelles were generated from the Poisson equation by assuming that all microtubules transport organelles. The expected probability of the microtubules with $0,1,2$, and 3 or more attached organelles is equal to $e^{-\lambda}$ and $\left(e^{-\lambda n}\right) / n !$, where $\lambda$ is the observed mean and $n$ is the number of attached organelles. In order to examine the distribution of microtubule-contacting organelles in neurites and growth cones, all the microtubules in 5 neurites and 4 growth cones were subdivided into segments, each $0.5 \mu \mathrm{m}$ long, and the number of microtubule-organelles contacts was determined for each segment. The expected numbers of contacts were calculated from the Poisson equation as described above. The goodness of fit of the expected and observed data was analyzed by the $\chi^{2}$ test.

\section{Results}

Organelles inside and outside of microtubule fascicles

Most of the organelles in neurites contacted microtubules (Fig. $1, A, B)$; most of these organelles $(91 \%$, Table 1$)$ were either small vesicles or large vacuoles. Vesicles were typically enclosed
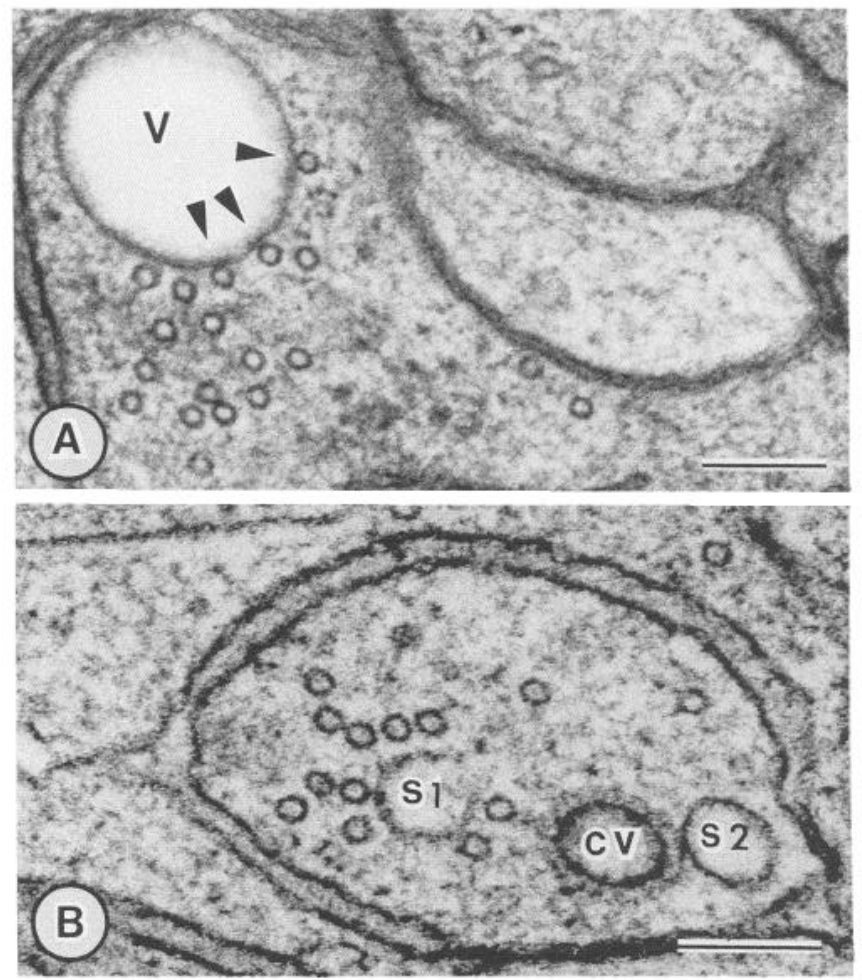

Figure 1. Electron micrographs of freeze-substituted tectal neurites showing contacts between a vacuole and a microtubule (arrows, $A$ ) and confinement of a vesicle within a microtubule fascicle $(B)$. Most small vesicles $(S 1, B)$ are confined within microtubule fascicles, but a few $(S 2$, $A)$ are not. Noncontacting vesicles $(S 2, B)$ are frequently located near the plasmalemma; coated vesicles $(\mathrm{CV})$ do not contact microtubules. Large vacuoles $(V, A)$ usually lie outside of microtubule fascicles but frequently contact several microtubules. Scale bar, $0.1 \mu \mathrm{m}$.

in a microtubule fascicle (Fig. $1 B$ ), whereas vacuoles and other organelles were usually at the periphery of a fascicle (Fig. $1 A$ ). To substantiate these morphological observations that vesicles (diameter $<150 \mathrm{~nm}$ ) and vacuoles (diameter $>150 \mathrm{~nm}$ ) in neurites were located in different axoplasmic subdomains, we measured the diameters of all vesicles and vacuoles ( 261 out of 381 ; Fig. 2) that were contacting microtubules in 5 reconstructed neurite segments, each about $10 \mu \mathrm{m}$ long. Almost all vesicles inside a fascicle were $<100 \mathrm{~nm}$ in diameter; in contrast, all vacuoles with larger diameters were arranged around the outside of the fascicle (Fig. 2).

Approximately $23 \%$ of the organelles ( 88 out of 381 ) in neurites, compared with only $1.5 \%$ (24 out of 1584$)$ in growth cones, were inside a microtubule fascicle, and most of these were in the most proximal part of the growth cone where the microtubule fascicles were still prominent.

\section{Distribution of microtubule-contacting organelles in growth cones}

Most microtubules in the neurites are in fascicles (Fig. 3A). There was no pattern we could see in the distribution of microtubule-contacting organelles along the $10 \mu \mathrm{m}$ reconstructed segments of microtubule (Fig. $3 B$ ). In the growth cones, however, microtubule fascicles typically splayed out along the proximo-distal axis and were not apparent in the distal growth cone (Fig. 4A). The appearance of individual microtubules also changed along the long axes of growth cones (Fig. 4B). More proximal individual microtubules appeared to be straight, while 


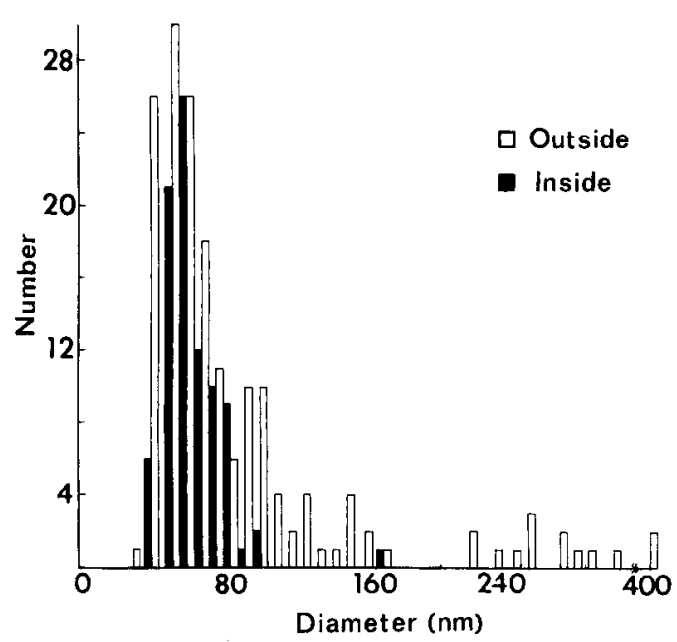

Figure 2. Diameters of organelles inside and outside of microtubule fascicles in 5 reconstructed neurite segments. Almost all of the vesicular organelles inside a fascicle are vesicles with diameters $<100 \mathrm{~nm}$, whereas the larger vacuolar organelles typically lie outside of a fascicle.

their distal extensions became undulated (Fig. $4 B$ ); these differences were consistently observed in all of the microtubules that extended into the distal regions of the 4 , reconstructed tectal growth cones (only 23 out of 89 microtubules extend to the distal regions). The frequency of vesicles contacting microtubules was greater in the straight microtubule subdomains (Fig. $4 B$ ). The straight subdomains of growth cone microtubules were confined to the more proximal region of the growth cone (Fig. $4 A$ ). The mean concentration of microtubule-contacting vesicles decreased from 16.1 per $\mu \mathrm{m}^{3}$ in the transition zones to 6.4 per $\mu \mathrm{m}^{3}$ in the proximal regions and further to 1.7 per $\mu \mathrm{m}^{3}$ in the distal region of the growth cone; all of these means were significantly lower than that in the neurites $\left(32.7\right.$ per $\mu \mathrm{m}^{3}$; Table 2 ). However, there were typically 15 microtubules in the proximal, but 10 or fewer microtubules in the distal, regions of growth cones compared with 11 microtubules in the neurites (Fig. 5). Thus, the concentration of microtubule-contacting vesicles was significantly lower, though the average number of microtubules was actually somewhat higher, in the proximal growth cone than in the neurites, meaning that the lower concentration of vesicles contacting microtubules in the proximal growth cone is not explained by a decrease in the number of microtubules.
In the more distal regions, the concentration of microtubulecontacting vesicles continued to decrease slightly (Fig. 5); this slight decrease is significant because individual growth cones showed the same tendency. However, this lower concentration of microtubule-contacting vesicles appears to parallel a decrease in the number of microtubules (Fig. 5, Table 2).

The concentration of vacuoles contacting microtubules appeared to be somewhat higher in the midregions of growth cones than either more distally or more proximally in the growth cone or axon (Fig. 5), but this is significant because similar distributions of microtubule-attached vaucoles were seen in every reconstructed growth cone. In any instance, there was clearly a systematic and continuous decrease in the absolute number of vesicles and vacuoles contacting microtubules along the proximo-distal axcs of growth cones (Fig. 5).

\section{Distribution of noncontacting organelles in growth cones}

In order to understand better the meaning of the low concentration of microtubule-contacting organelles in the more distal regions of the chick tectal growth cones, we also examined distributions of vesicles and vacuoles that were not contacting microtubules along the proximo-distal axis. Most of the noncontacting organelles in the most proximal parts of growth cones were located near microtubules, but most of those in the midand more distal regions were further away from microtubules (Fig. 6). In agreement with these morphological observations were the data obtained by measuring the shortest distance between each noncontacting vesicle or vacuole and the nearest microtubules in the 4 reconstructed growth cones (Fig. 7). The mean distances between these noncontacting organelles and microtubules increased progressively in the proximo-distal dircction, reaching a maximum of approximately $600 \mathrm{~nm}$ in the midregions of growth cones (Fig. 7). In contrast, the mean distances between the noncontacting organelles and microtubules were significantly less in the distal than in the middle growth cones. However, the SDs around these mean distances were large, suggesting that organelles in the distal regions are not organized around the microtubules there.

Only $11 \%$ of the organelles in growth cones contacted microtubules, in contrast to $75 \%$ of those in neurites (Table 1). Most of the noncontacting organelles (83\%) were concentrated in the distal region of growth cones (Fig. 8, Table 2), in contrast to their homogeneous distribution within the 5 reconstructed neurite segments (Fig. 8). This finding, along with the measurements

Table 1. Relative frequency of different types of organelles and percentage of each type contacting one or more microtubules (MT)

\begin{tabular}{llllll} 
& \multicolumn{2}{l}{ Neurite $^{a}$} & & & \multicolumn{2}{l}{\begin{tabular}{l} 
Growth cone \\
\cline { 3 - 5 }
\end{tabular}} & $\begin{array}{l}\text { Frequency } \\
\text { Organelle-type }\end{array}$ & $\begin{array}{l}\text { Percentage } \\
\text { contacting }\end{array}$ & & $\begin{array}{l}\text { Frequency } \\
(\%)\end{array}$ & $\begin{array}{l}\text { Percentage } \\
\text { contacting } \\
\text { MT }\end{array}$ \\
\hline Small vesicles & 85.3 & 75 & 80.5 & 11 \\
Flat vacuoles & 3.9 & 40 & 6.7 & 17 \\
Large vacuoles & 5.8 & 77 & 2.9 & 29 \\
Mitochondria & 1.8 & 86 & 1.8 & 27 \\
Coated vesicles & 1.2 & 0 & 3.8 & 0 \\
Single disks & 2.0 & 0 & 4.0 & 9 \\
Stacked disks & 0 & 0 & 0.3 & 0 \\
\hline
\end{tabular}

"A total of 381 organelles in 5 neurite segments and 1584 organelles in 4 entire growth cones were counted. 


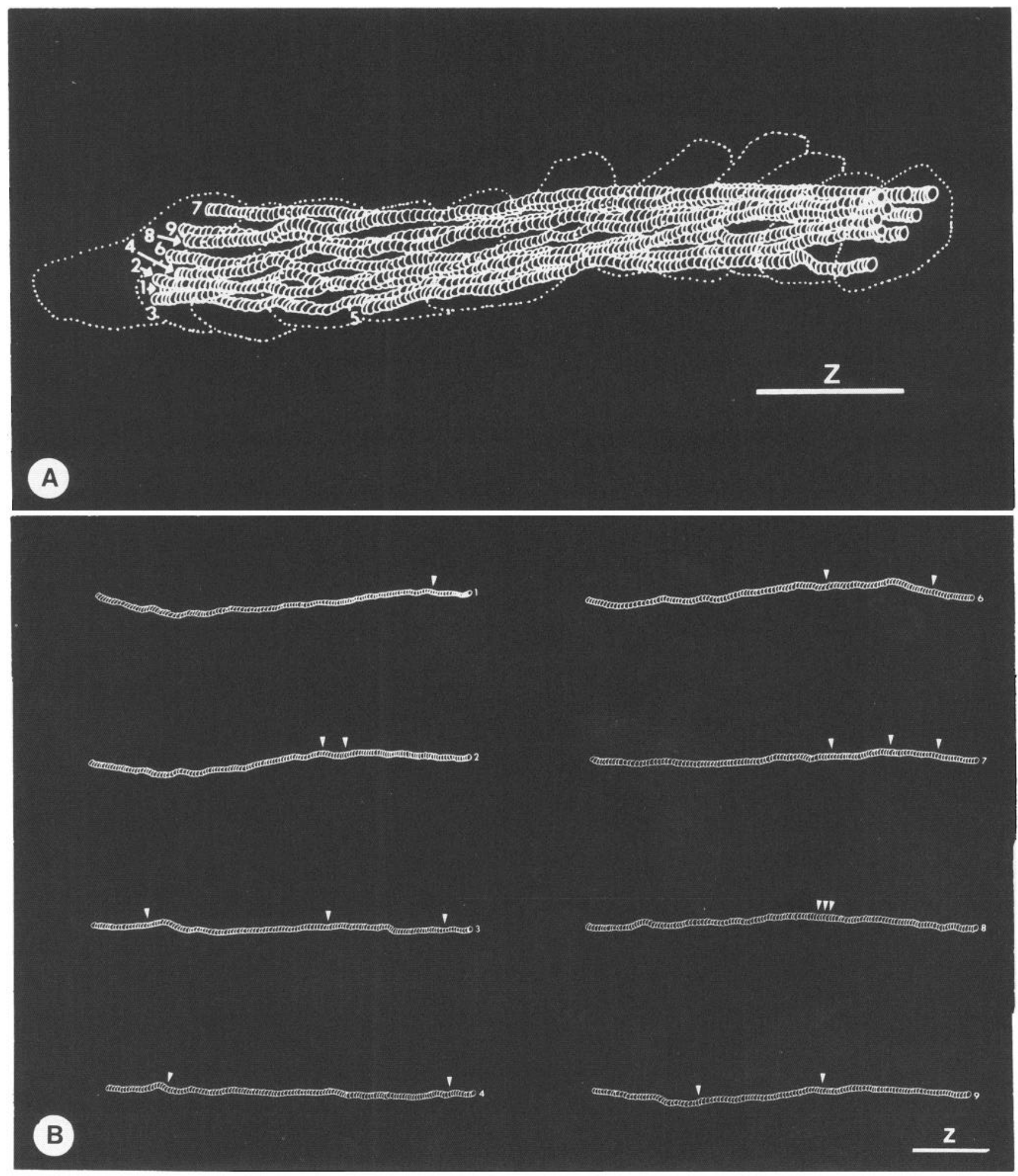

Figure 3. Serial reconstruction of microtubules and distribution of microtubule-contacting organelles within a $10 \mu \mathrm{m}$ neurite segment. A fascicle of microtubules is shown in its neurite $(A)$. Microtubule-contacting organelles, as indicated by small arrowheads on individual microtubules $(B)$ from the fascicle in $A$ are located within or along microtubules in the fascicle. Some microtubules are not contacted by organelles (e.g., microtubule number 5 in $A$ ), but there are typically $2-3$ microtubule-contacting organelles on each $10 \mu \mathrm{m}$ segment of microtubules. The 3 arrowheads on microtubule 8 indicate a mitochondrion making several contacts with it. The $Z$-bar corresponds to $2 \mu \mathrm{m}$ along the longitudinal axis of growth.

of mean distances between organelles and microtubules (Fig. 7), give us a basis to calculate an association tendency between organelles and microtubules (the ratio of the concentration of microtubule-contacting organelles to the concentration of non- contacting organelles times the concentration of microtubule segments not contacted by organelles). A low association number between organelles and microtubules would be another indication of their relative low mutual affinity. The association 


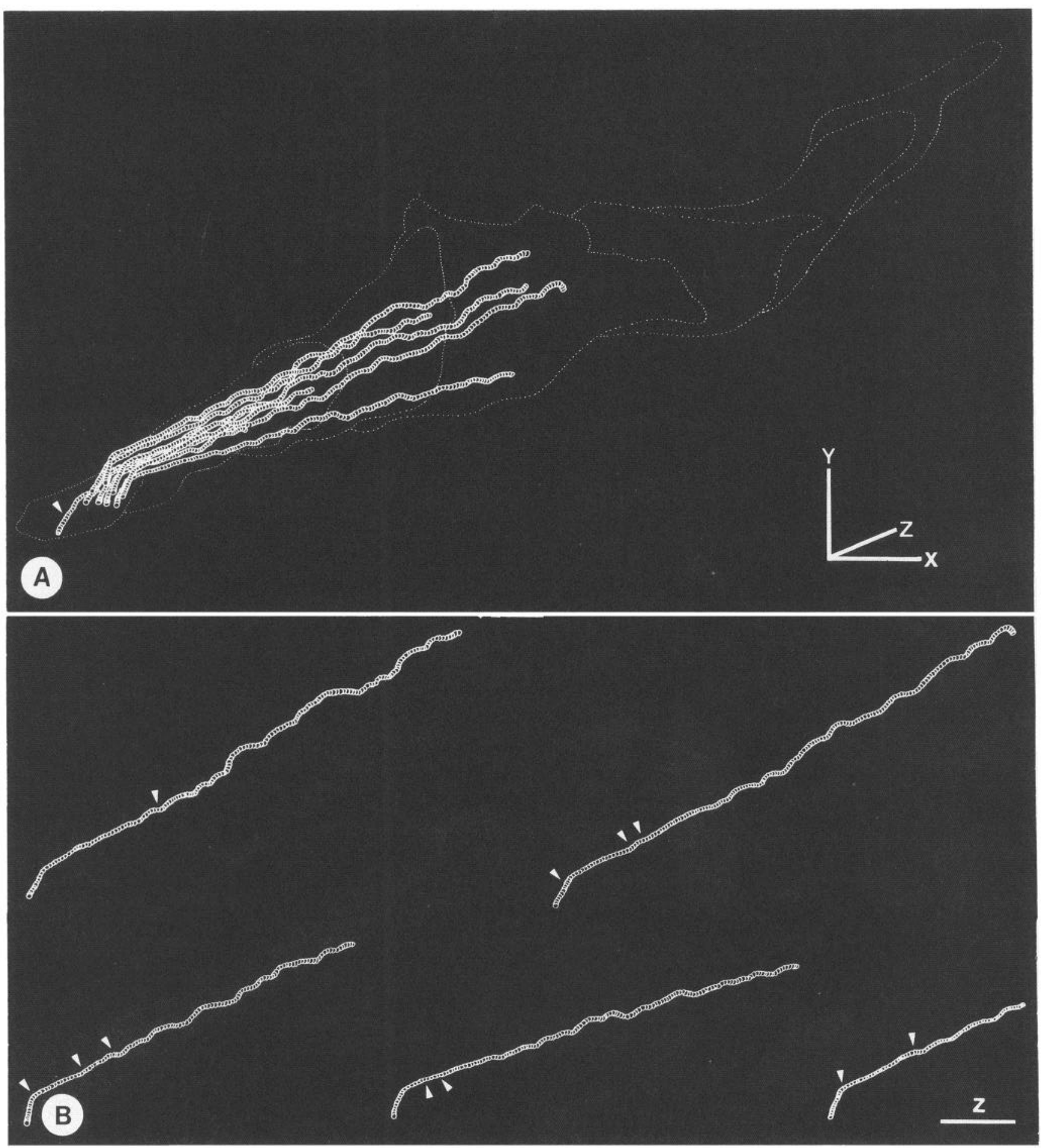

Figure 4. Serial reconstruction of microtubules and distribution of microtubule-contacting organelles (arrowheads) in a tectal growth cone (dotted outline). Microtubule fascicles typically splay out along the proximo-distal axis of the freeze-substituted growth cone $(A)$. Most microtubules terminate in the proximal region of the growth cone, but a few (not shown here) extend to the tip of the filopodium. Microtubule-contacting organelles are not uniformly distributed along microtubules in the growth cone (as shown individually in $B$ ) but are concentrated proximally in the growth cone. The microtubule-contacting organelles appear to be restricted to the proximal straight subdomains of the microtubules but excluded from the more distal undulating subdomains in the growth cone. The $X$-and $Y$-bars, representing the cross-sectional $X$-and $Y$-axes, indicate $0.5 \mu$ m, while the $Z$-bar representing the proximal-distal axis indicates $2 \mu \mathrm{m}$.

tendency between vesicles and microtubules in the transition zone of the growth cone was twice as high as in neurites $(5.9 \mathrm{vs}$ 2.7; Table 2), but was 3 times lower in the proximal regions (1.0); in contrast, association ratios of vacuoles to microtubules were higher ( 3.2 proximally and 3.0 distally) in the growth cone than in the neurites (2.3), which means that the percentage of microtubule-contacting vacuoles $(46 \%)$ is higher than that of vesicles $(18 \%)$ in the proximal growth cone, although it is similar 
in neurites ( $75 \%$ for vesicles and $77 \%$ for vacuoles). In comparison, the association tendency for vesicles $(0.1)$ and for vacuoles (0.3) was lowest in the distal regions, reflecting the fact that fewer organelles were attached to microtubules there, even though the local concentration of vesicles was much higher and that of vacuoles was slightly higher than more proximally (Table 2). Therefore, the concentrations of organelles and microtubules cannot be the only factors determining the frequencies of contacts between organelles and microtubules.

\section{Probability of organelles making contacts with microtubules}

In order to determine whether the high concentration of noncontacting organelles in the distal regions and of microtubulecontacting organelles in neurites and the most proximal parts of growth cones can be explained by the presence of a subclass of microtubules incapable of binding organelles, we analyzed the observed and expected frequencies of microtubule-contacting vesicles and vacuoles in neurites and growth cones. The observed frequency of organelle-microtubule contacts in the neurites and the transition zones of the 4 reconstructed growth cones did not vary significantly from the expected frequency calculated from the Poisson distribution generated by the assumption that organelles making contacts with each of the 0.5 $\mu \mathrm{m}$ segments of microtubules along the long axis are independent events and that all microtubules can bind organelles (see Materials and Methods; Fig. 9). This result suggests that all neuritic microtubules are equally able to bind organelles. In contrast, the observed frequencies of vesicle- and vacuole-microtubulc contacts in the proximal and the distal regions were significantly different from those predicted, only 47 and $41 \%$ of their expected frequencies (Fig. 9). Similarly, the observed frequencies of microtubule segments that were not in contact with organelles in the more distal region of the growth cones were significantly higher than the expected frequencies (Fig. 9). These results suggest either that some growth cone microtubules are

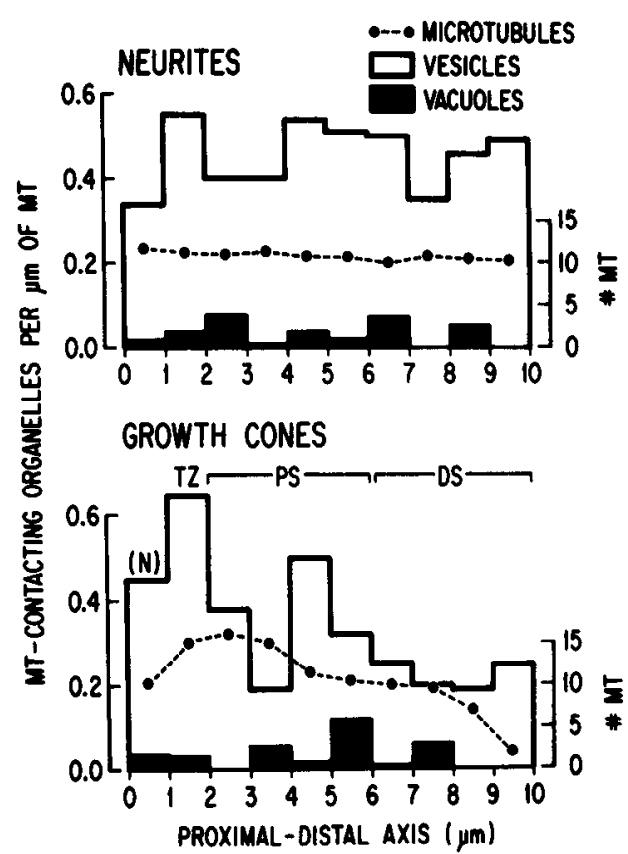

Figure 5. Average numbers of vesicles (empty bars) and vacuoles (solid bars) contacting successive $1 \mu \mathrm{m}$ microtubule subsegments along the longitudinal axes of reconstructed neurites (top) and growth cones (bottom). The superimposed dotted line indicates the average number of microtubules in successive $1 \mu \mathrm{m}$ subscgments. Microtubulc-contacting vesicles and vacuoles are uniformly distributed throughout the reconstructed neurite segments (top). In contrast, the number of microtubuleconlacting vesicles per micron of microtubule decreases abruptly in the most proximal regions $(P S)$ of the growth cones (bottom) and continues to decrease distally. However, microtubule-contacting vacuoles appear to increase slightly and to decrease distally, which is still proximal to where the microtubules end. Pooled data from 5 neurites, each approximately $10 \mu \mathrm{m}$ long and from 4 entire growth cones, each approximately $15-20 \mu \mathrm{m}$ long. The transition zones $(T Z)$, the proximal $(P S)$, and the distal regions $(D S)$ are defined by structural characteristics previously defined (Cheng and Reese, 1987).

\begin{tabular}{|c|c|c|c|c|c|}
\hline Parameters & Neurite & Growth cone & $\mathrm{TZ}$ & PS & DS \\
\hline MT segments ${ }^{t}$ & 216 & 226 & 30 & 110 & 86 \\
\hline Vesicles & 65 & 318.7 & 14.5 & 102 & 202.2 \\
\hline Vacuoles & 4.4 & 11.6 & 0.8 & 5.0 & 5.8 \\
\hline Volume $\left(\mu \mathrm{m}^{3}\right)$ & 1.5 & 8.2 & 0.6 & 3.9 & 3.7 \\
\hline $\mathrm{MT} / \mu \mathrm{m}^{3}$ & 144.0 & 27.5 & 50.0 & 28.2 & 23.2 \\
\hline \multicolumn{6}{|l|}{ Vesicles $/ \mu \mathrm{m}^{3}$} \\
\hline Attached & 32.7 & 4.26 & 16.1 & 4.8 & 1.7 \\
\hline Not attached & 10.8 & 34.61 & 8.0 & 21.3 & 52.9 \\
\hline \multicolumn{6}{|l|}{ Vacuoles $/ \mu \mathrm{m}^{3}$} \\
\hline Attached & 2.3 & 0.4 & 0.8 & 0.58 & 0.13 \\
\hline Not attached & 0.7 & 1.01 & 0.5 & 0.69 & 1.43 \\
\hline \multicolumn{6}{|c|}{ Association tendency ${ }^{\prime}$} \\
\hline Vesicle & 2.7 & 0.5 & 5.9 & 1.0 & 0.2 \\
\hline Vacuole & 2.3 & 1.5 & 3.2 & 3.0 & 0.3 \\
\hline
\end{tabular}

\footnotetext{
"Number of $0.5-\mu \mathrm{m}$-long microtubule segments per $\mu \mathrm{m}^{3}$.

All figures are averaged from 5 neurite segments and 4 entire growth cones.

Growth cones here are separated into 3 structurally defined domains: transition (TZ), the proximal (PS), and distal (DS) regions.

"Microtubules were arbitrarily subdivided into $0.5-\mu \mathrm{m}$-long microtubule segments.

Ratio of concentration of microtubule-contacting organelles to the product of concentrations of noncontacting organelles and MT segments not in contact with organelles.
} 

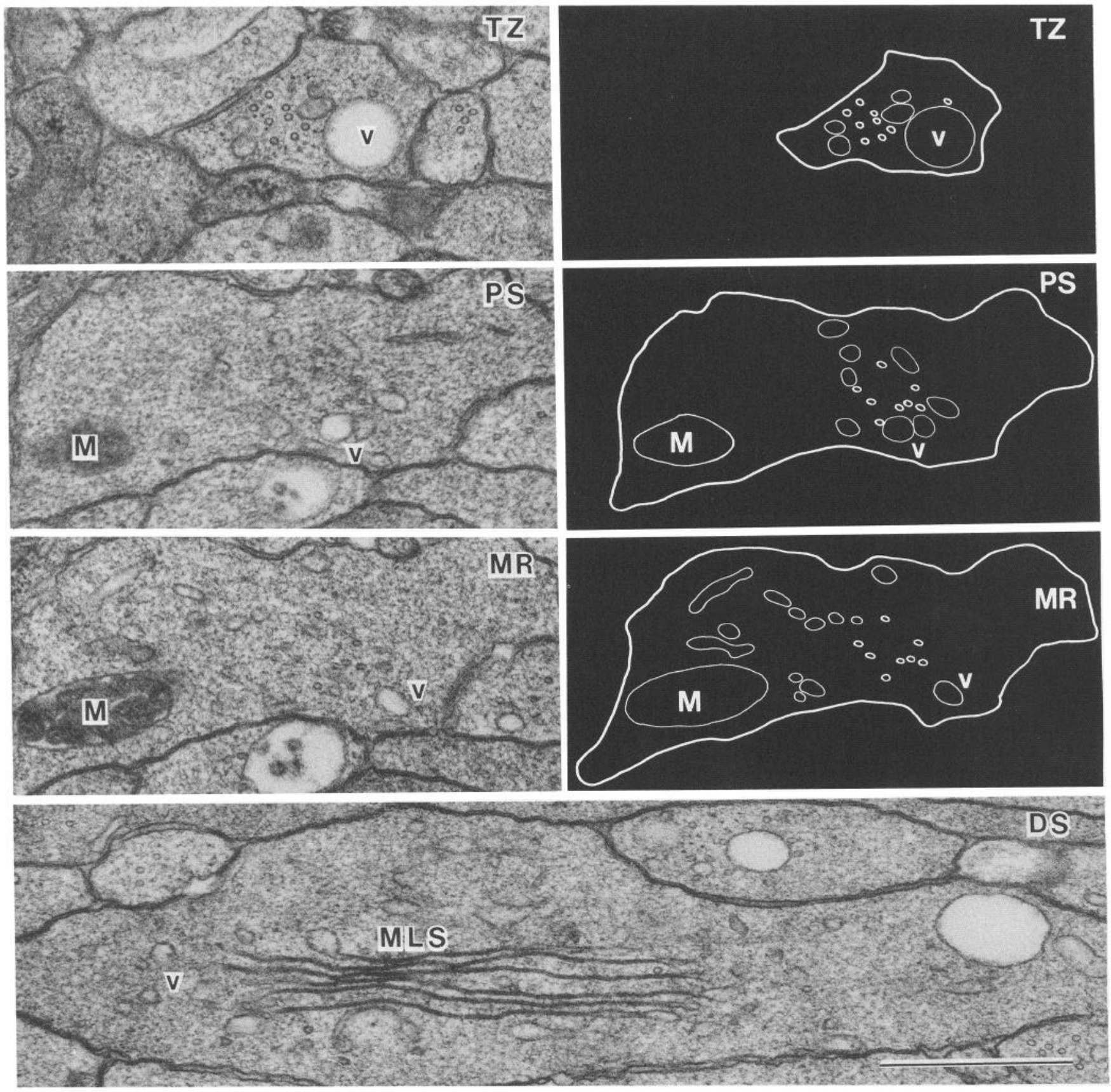

Figure 6. Distribution of noncontacting organelles and microtubules in a serially sectioned growth cone. Vesicles and vacuoles (v) in the transition zone $(T Z)$ and the proximal region $(P S)$ are close to a fascicle of microtubules. However, most organelles in the midregion $(M R)$ are further away from microtubules in the fascicle; the fascicle also begins to splay out (see tracings of the micrographs). In the distal regions (DS), microtubules do not form bundles, and organelles do not appear to have any apparent relationship to microtubules. Various organelles ( $v$ ) are, instead, associated with stacks of lumenless membrane disks $(M L S)$, which are typical of the distal growth cone. Mitochondria $(M)$ in growth cones typically lie farther away from microtubule fascicles than the other organelles. Scale bar, $0.5 \mu \mathrm{m}$.

at least transiently unable to bind organelles or that other factors, such as binding organelles to other cytoplasmic components, discourage their binding to growth cone microtubules.

\section{Discussion}

Neuritic subdomains for anterograde and retrograde transport Microtubules in the neurites from intact chick optic tecta form fascicles (see also Cheng and Reese, 1985a), and membranelimited organelles lie within, as well as around, these fascicles. Vesicles with diameters $<100 \mathrm{~nm}$ typically lie inside a fascicle, whereas other organelles with larger diameters are arrayed at its periphery. Thus, it appears that the microtubule-associated axoplasmic domains (Schnapp and Reese, 1982) in the neurites have separate channels or subdomains defined by the preferred position of the small and large organelles in relation to the microtubule fascicles.

In some axons, organelle diameter appears to be indicative of the direction of movement, with smaller organelles moving away from the cell body (Smith et al., 1975; Tsukita and Ishikawa, 1980; Miller and Lasek, 1985). The diameters of vacuoles 


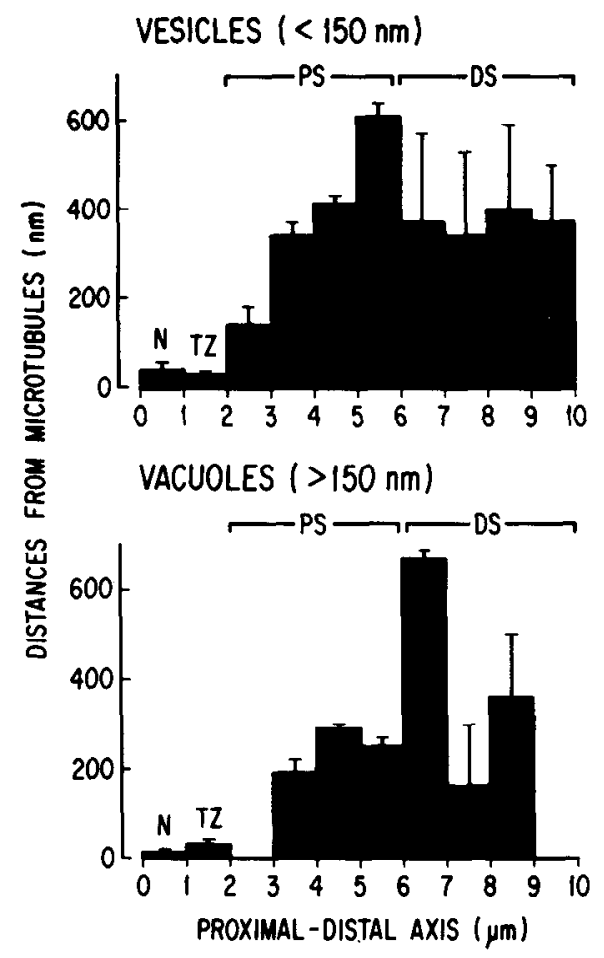

Figure 7. Separations between vesicles (top) or vacuoles (bottom) and microtubules along the longitudinal axes of growth cones. Mean distances of noncontacting organelles from the closest microtubule are typically 10 -fold greater in the varicosities of growth cones than in neurites $(N)$. The mean distances from the nearest microtubule increase systematically from the transition 70 e $(T 7)$ to the proximal region $(P S)$, reaching a maximum of approximately $600 \mathrm{~nm}$ in the mid-region of growth cones. The maximum separation of vacuoles from the nearest microtubule appears to be approximatcly $1 \mu \mathrm{m}$. The mean distances of both vesicles and vacuoles from microtubules are uniformly less in the distal regions $(D S)$ than in the midregions; however, the variances of these distances are much greater in the distal growth cones than more proximally.

located outside a microtubule fascicle in intact neurites from developing chick optic tecta are similar to those of retrogradely translocating organelles in extruded squid axoplasm, whereas those of vesicles inside a fascicle are similar to those in anterograde transport (Miller and Lasek, 1985; see also introductory remarks above). Therefore, the cytoplasmic subdomains around the periphery of microtubule fascicles in the neurites may support retrograde transport, whereas the central cores of the fascicles may support anterograde transport.

Fascicles of microtubules define compartments rich in both microtubules and organelles in the core of the chick tectal neurites. This increase in the effective concentration of microtubules and organelles could increase the probability of contacts between organelles and microtubules (Vale et al., 1985a). Cocompartmentalization of microtubules and organelles would also favor multiple binding between organelles and microtubules; indeed, most organelles within a fascicle contact one or more microtubules. Thus, the arrangement of fascicles of neuritic microtubules ensures high local coconcentrations of microtubules and organelles to promote attachment of these organelles to microtubules.

\section{Unloading and loading of organelles onto microtubules}

The subdomains defined by microtubule fascicles in neurites are inconspicuous in growth cones, due in part to splaying of

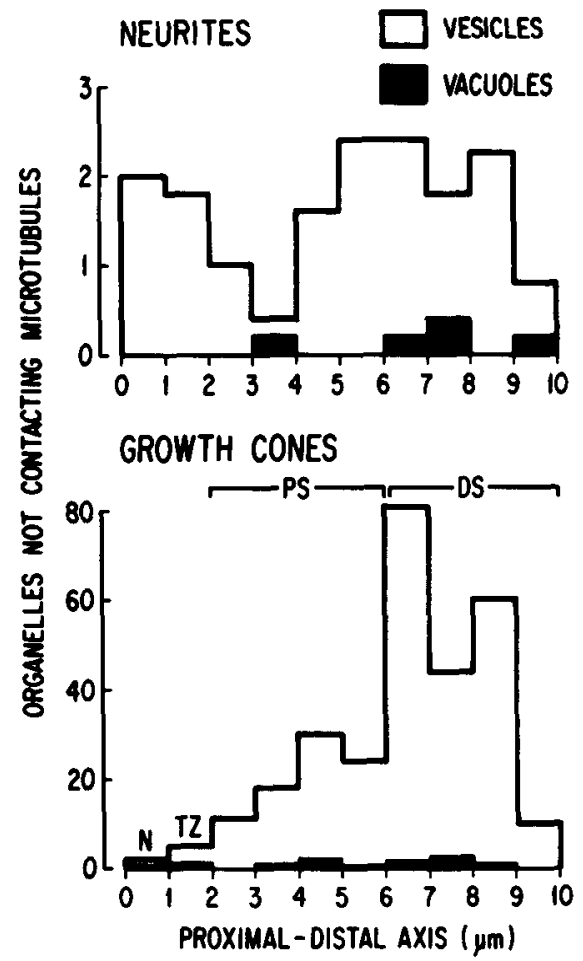

Figure 8. Average numbers of vesicles (empty bars) and vacuoles (solid bars) not contacting microtubules in successive $1-\mu \mathrm{m}$-long subsegments of neurites (top) or growth cones (bottom). No specific patterns in the distribution of noncontacting vesicles and vacuoles are observed in the 5 reconstructed neurites. In contrast, the number of noncontacting vesicles increases progressively in the proximal regions (PS) and even more in the distal regions $(D S)$ of the 4 reconstructed growth cones; the number of noncontacting vacuoles may also increase slightly more distally.

microtubule fascicles and, in part, to a progressive decrease in the total number of microtubules along the proximo-distal axis. Although vesicles and vacuoles are still clustered close to the periphery of microtubule fascicles in the most proximal parts of growth cones, they become less frequent distally where the fascicles splay out. Vesicles contacting microtubules, which presumably represent those in fast transport (Miller and Lasek, 1985), disappear progressively and then precipitously along the proximo-distal axis of the growth cone. Also, the distance of noncontacting vesicles from microtubules increases systematically along the proximo-distal axis, reaching a maximum in the growth cone midregions. Together, these findings suggest that vesicles moving into growth cones by fast anterograde transport have a progressively increased probability of coming off microtubules. Indeed, most of the vesicles in growth cones are not contacting microtubules and most of these organelles are concentrated in the distal regions. Thus, we can infer that vesicles in fast anterograde transport are unloaded in the proximal rather than in the distal regions of the growth cone. Although termination of microtubules in a growth cone fascicle may be partially responsible for unloading of vesicles, splaying of microtubules in the fascicle may also play a significant role because the concentration of vesicles contacting microtubules is 6 times greater in the neurites and 3 times greater in the transition zones (where fascicles are still conspicuous) than in the proximal regions (where fascicles splay out), even though the average number of microtubules in these regions differs only slightly. 

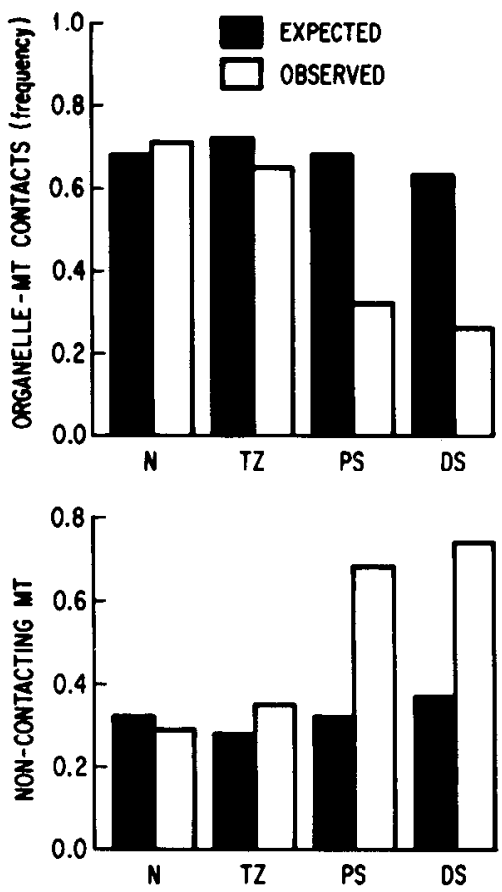

Figure 9. Comparison between the expected and observed frequency of organelle-microtubule contacts along neurite and growth cone microtubules calculated from Poisson distributions based on their overall frequency and on the assumption that every microtubule is capable of transporting organelles (see Materials and Methods). The differences between the expected and observed frequency of organelle-microtubule contacts in the proximal (PS) and distal (DS) regions are significant, but those in the neurites $(N)$ and the transition zones $(T Z)$ are not, as detcrmincd by $\chi$ ' test. This result suggests that every microtubule in neurites and the most proximal growth cone can transport organelles but that some (or perhaps all) microtubules in the more distal growth cone have a lower affinity for organelles.

In the more distal regions, however, the decrease in number as well as the absence of microtubule fascicles may not account entirely for the low concentration of microtubule-contacting vesicles and the high concentration of noncontacting vesicles. Other undefined factors may promote dissociation of attached organelles moving in the anterograde direction, but it is not known whether kinesin or other factors promoting organelle transport (Vale et al., 1985b-d) are less concentrated in the distal than the proximal regions, nor can an important role for the association of organelles with each other or with other cytoplasmic elements be discounted. It is also possible that binding between organelles and microtubules is inhibited by proteins or other factors concentrated in the distal regions of growth cones.

The straightness of individual microtubules in a fascicle appears to be correlated with a role in the unloading of vesicles in anterograde transport. Most of the microtubule-contacting organelles in growth cones are attached to the straight segments of individual microtubules; in contrast, they are rare along the more distal undulating segments. The straight segments of microtubules are positioned in the most proximal parts of growth cones (where vesicles in transport are thought to begin unloading) compared with the undulated segments positioned more distally (where most of these vesicles are evidently detached from the microtubules). Thus, the vesicles moving anterogradely along microtubules into the growth cones appear to be unloaded preferentially at and after the points where microtubules assume an undulating shape. Our conclusion that unloading of anter- ogradely moving organelles occurs primarily in the midregions of growth cones is supported by our observations that microtubule-contacting organelles assumed to be transported along microtubules are prevalent only in the most proximal growth cones, and that the tendency of organelles to associate with microtubules is considerably higher in neurites and the most proximal growth cone than more distally.

Large vacuoles attached to microtubules in growth cones are thought to be in retrograde transport because large-diameter organelles in the squid and other axons are transported retrogradely, towards the cell bodies (Smith, 1980; Miller and Lasek, 1985), and because endocytotic vacuoles are transported retrogradely out of mature nerve endings (Berlinrood et al., 1972; Breuer et al., 1975; Stoekel and Thoenen, 1975; Bunge, 1977). Thus, vacuoles contacting microtubules in growth cones are taken to be organelles in retrograde transport (Tsukita and Ishikawa, 1980).

Attachment of vacuoles to microtubules to initiate retrograde transport also appears to occur in the midregions of growth cones because the concentration of microtubule-contacting vacuoles, presumably representing those in fast retrograde transport (Smith, 1980; Miller and Lasek, 1985; Schnapp et al., 1985), is somewhat higher in the midregions of growth cones than either both more proximally or distally. In addition, the mean distances between vacuoles and microtubules in the tectal growth cones are significantly less more proximally, perhaps reflecting a higher tendency of vacuoles to associate with microtubules in the proximal midregions than more distally; also, the ratio of microtubule-attached to unattached vacuoles is significantly higher in the neurites and the proximal regions than more distally. The small, but significant, increase in vacuole concentration in the midregion of the growth cone could mean that vacuoles attaching to microtubules in preparation for retrograde transport pause before beginning to move retrogradely out of the midregion of the growth cone.

Thus, a loading zone for organelles destined for retrograde transport appears to overlap with an unloading zone for organelles in anterograde transport; both zones appear to be located within the midregions of growth cones. The straight and undulating segments of a growth cone microtubule appear to coincide roughly with the growth cone regions where organelles are introduced to or removed from fast axonal transport. It therefore appears that there are separate and distinctive regions along the microtubules in the chick tectal growth cones designated for terminating anterograde and for initiating retrograde transport.

\section{Lack of organelle association with microtubules in the distal growth cone}

In the distal regions, microtubules generally are not in fascicles, and all of the microtubules originating from neurites are undulated. Also, microtubules are least concentrated and noncontacting organelles are most concentrated in the distal regions of growth cones. In parallel with these distinctive structural features, the functional role of most orgarielles in the distal regions also appears to be different from that in the proximal regions. There is no apparent organized relationship between microtubules and organelles in the distal growth cones, as suggested by the high variability and lack of pattern in the distances between organelles and microtubules. In fact, most of the noncontacting vesicles and vacuoles in the distal growth cones do not appear to represent a pool of organelles which are about to bind to or 
have just dissociated from microtubules because they are not selectively distributed near or around microtubules. They are, instead, closely associated with stacks of lumenless membrane disks, which are typical of the distal regions of growth cones (see also Cheng and Reese, 1985a); these vesicles and vacuoles are thought to have a role in providing membrane for addition to the growth cone plasmalemma during filopodial extension (Cheng and Reese, 1987). The high concentrations of noncontacting vesicles and vacuoles in the distal regions are consistent with the idea that they are involved in local recycling of the plasmalemma during filopodial extension or retraction (Cheng and Reese, 1987). This idea is also supported by the observation that noncontacting vesicles and vacuoles are, in part, derived from coated vesicles originating from the growth cone plasmalemma (Cheng and Reese, 1987).

Our current working hypothesis is that most of the vesicles arriving at the growth cones by fast anterograde transport are detached and added to a pool of organelles involved in recycling of the local plasmalemma (Cheng and Reese, 1987). Similarly, some of the endocytotic vacuoles destined for fast retrograde transport (Cheng and Reese, 1987) are withdrawn from the distal pool of membrane to carry specific materials back to the cell body. However, it is not clear how these vesicles and vacuoles, which are similar in appearance but different in function, are specified for either fast axonal transport or for local recycling with the growth cone plasmalemma.

\section{References}

Allen, R. D. (1975) Evidence for firm linkages between microtubules and membrane-bound vesicles. J. Cell Biol. 64: 497-503.

Allen, R. D., J. Metuzals, I. Tasaki, S. T. Brady, and S. P. Gilbert (1982) Fast axonal transport in squid giant axon. Science 218: $1127-$ 1128.

Berlinrood, M., S. M. McGee-Russell, and R. D. Allen (1972) Pattern of particle movement in nerve fibres in vitro-an analysis by photokymography and microscopy. J. Cell Sci. 11: 875-886.

Brady, S. T., R. J. Lasek, and R. D. Allen (1982) Fast axonal transport from squid giant axon. Science 218: 1129-1131.

Bray, D., C. Thomas, and G. Shaw (1978) Growth cone formation in cultures of sensory neurons. Proc. Natl. Acad. Sci. ISA 75: 52265229.

Breuer, A. C., C. N. Christian, M. Henkart, and P. G. Nelson (1975) Computer analysis of organelle translocation in primary neuronal cultures and continuous cell lines. J. Cell Biol. 65: 562-576.

Bunge, M. B. (1977) Initial endocytosis of peroxidase or ferritin by growth cones of culture nerve cells. J. Neurocytol. $6: 407-437$.

Cheng, T. P. O., and T. S. Reese (1985a) Polarized compartmentalization of cytoplasmic organelles in chick tectal growth cones. J. Cell Biol. 101: 1437-1480.

Cheng, T. P. O., and T. S. Reese (1985b) Membranes recycle at chick tectal growth cones. J. Cell Biol. 101(No. 5, Pt. 2): 290a.

Cheng, T. P. O., and T. S. Reese (1987) Recycling of plasmalemma in chick tectal growth cones. J. Neurosci. 7: 1752-1759.

Conper, P. D., and R. S. Smith (1974) The movement of optically detectable organelles in myelinated axons of Xenopus laevis. J. Physiol. (Lond.) 242: 77-97.

Feldman, E. L., D. Axelrod, M. Schwartz, A. M. Heacock, and B. W. Agranoff (1981) Studies on the localization of newly added membranes in growing neurites. J. Neurobiol. 12: 591-598.

Hayden, J. H., R. D. Allen, and R. D. Goldstein (1983) Cytoplasmic transport in keratocytes: Direct visualization of particles translocation along microtubules. Cell Motility 3: 1-9.

Hendry, I. A., K. Stockel, H. Thoenen, and L. L. Iversen (1974) The retrograde axonal transport of nerve growth factor. Brain Res. 68: 103-121.

Kachar, B., P. C. Bridgman, and T. S. Reese (1988) Dynamic shape changes of cytoplasmic organelles translocating along microtubules. J. Cell Biol. (in press).

Kristenson, K. (1977) Retrograde axonal transport of horseradish peroxidase uptake at mouse neuromuscular junction following systematic injection. Acta Neuropathol. 38: 143-147.

Kristenson, K., and Y. Olsson (1971) Retrograde axonal transport of protein. Brain Res. 29: 363-365.

LaVail, J. H., and M. M. LaVail (1974) The retrograde intraaxonal transport of horseradish peroxidase in the chick visual system: A light and electron microscopic study. J. Comp. Neurol. 157: 303-358.

Leestma, J. E. (1976) Velocity measurement of particulate neuroplasmic flow in organized CNS tissue cultures. J. Neurobiol. 7: 173183.

Martz, D., R. J. Lasek, S. T. Brady, and R. D. Allen (1984) Mitochondrial movement in axons: Membranous organelles may interact with the force generating system through multiple surface binding sites. Cell Motility 4: 89-101.

Miller, R. T., and R. J. Lasek (1985) Cross-bridges mediate anterograde and retrograde vesicle transport along microtubules in squid axoplasm. J. Cell Biol. 101: 2181-2193.

Pfenninger, K. H., and M. Maylie-Pfenninger (1981) I. Regional distribution of surface glycoconjugates. J. Cell Biol. 89: 536-546.

Raine, C. S., B. Ghetti, and M. F. Shelanski (1971) On the association between microtubules and mitochondria within axons. Brain Res. 34: 389-393.

Schnapp, B. J., and T. S. Reese (1982) Cytoplasmic structure in rapid frozen axons. J. Cell Biol. 94: 667-679.

Schnapp, B. J., R. D. Vale, M. P. Sheetz, and T. S. Reese (1985) Single microtubules from squid axoplasm support bidirectional movement of organelles. Cell 40: 455-465.

Schroer, T. A., S. T. Brady, and R. B. Kelly (1985) Fast axonal transport of foreign synaptic vesicles in squid axoplasm. J. Cell Biol. 101: 568-572.

Smith, R. S. (1980) The short term accumulation of axonally-transported organelles in the region of localized lesions of single myelinated axons. J. Neurocytol. 9: 39-65.

Smith, D. S., U. Jarlfors, and B. F. Cameron (1975) Morphological evidence for the participation of microtubules in axonal transport. Ann. NY Acad. Sci. 253: 472-506.

Stoekel, K., and H. Thoenen (1975) Retrograde axonal transport of nerve growth factor: Specificity and biological importance. Brain Res. 85: 337-341.

Tsukita, S., and H. Ishikawa (1980) The movement of membranous organelles in axons. Electron microscopic identification of anterogradely and retrogradely transported organelles. J. Cell Biol. 84: 513 530.

Vale, R. D., B. J. Schnapp, T. S. Reese, and M. P. Sheetz (1985a) Movement of organelles along filaments dissociated from the axoplasm of the squid giant axon. Cell 40: 449-454.

Vale, R. D., T. S. Reese, and M. P. Sheetz (1985b) Identification of a novel force-generating protein, kinesin, involved in microtubulebased motility. Cell 42: 39-50.

Vale, R. D., B. J. Schnapp, T. Mitchison, E. Steuer, T. S. Reese, and M. P. Sheetz (1985c) Different axoplasmic proteins generate movement in opposite directions along microtubules in vitro. Cell 43:623632.

Vale, R. D., B. J. Schnapp, T. S. Reese, and M. P. Sheetz (1985d) Organelle, bead, and microtubule translocations promoted by soluble factors from the squid giant axon. Cell 40: 559-569. 